\title{
Recomendaciones para el diagnóstico y tratamiento antimicrobiano de la neumonía bacteriana adquirida en la comunidad en pediatría
}

\author{
José Cofré, Daniela Pavez², Regina Pérez ${ }^{3}$ y Jaime Rodríguez ${ }^{4}$ en representación \\ del Comité de Antimicrobianos, Sociedad Chilena de Infectología. 2019
}

\section{Recommendations for the diagnosis and antimicrobial treatment of bacterial community acquired pneumonia in pediatrics}

Keywords: Community acquired pneumonia; pediatrics; etiological diagnosis; empirical treatment.

Palabras clave: Neumonía adquirida en la comunidad: pediatría; diagnóstico etiológico; tratamiento empírico.

\section{Introducción}

E 1 progreso socio-económico de la población chilena se ha traducido también en la evolución epidemiológica de las neumonías en pediatría. De ser una primordial causa de mortalidad, junto con la diarrea aguda, hoy, la neumonía continúa siendo una importante causa de hospitalización, pero su letalidad se ha reducido considerablemente. Factores determinantes en este progreso han sido: mejores condiciones nutricionales de los pacientes, mejor y pronto acceso a la salud, optimización de los servicios de urgencia periféricos y aquellos adosados a los hospitales con servicios pediátricos, avances en los cuidados intensivos de pacientes críticos $\mathrm{e}$, indudablemente, cambios etiológicos, a saber, bacterias por virus como primerísima etiología de las IRAS bajas. Este último componente- la evolución etiológica de la neumonía adquirida en la comunidad por niños-es abordado en esta guía, cuyo objeto es racionalizar el uso prudente y adecuado de antimicrobianos en la neumonía de causa bacteriana en Pediatría

Esta guía excluye aquellas neumonías que afectan a pacientes con afecciones crónicas (que mantienen contacto estrecho con recintos hospitalarios y/o con internaciones periódicas), pacientes inmunocomprometidos y lactantes bajo 3 meses de edad. Diversos factores (ambientales, comorbilidades, inmunitarios, etc.), nos llevan a no incluir a estos pacientes en una recomendación general.

\section{Definiciones}

- Neumonía/bronconeumonía: infección/inflamación pulmonar que compromete, en grado variable, los alvéolos pulmonares, el espacio intersticial pulmonar y los bronquíolos.

- Neumonía bacteriana: aquel proceso neumónico en que se documenta la presencia de una bacteria en el pulmón (mediante punción pulmonar), secreción bronquial o en un fluido normalmente estéril (sangre, líquido pleural) o una respuesta inmunológica contra un patógeno respiratorio (anticuerpos específicos tipo IgM o curva ascendente de IgG). Las más de las veces la etiología bacteriana es un supuesto, considerando la dificultad en disponer de muestras de tejido pulmonar o secreción bronquial y el bajo rendimiento del estudio del cultivo bacteriológico de sangre y efusión pleural (Ver párrafo: Cuándo una NAC es o puede ser de etiología bacteriana).

- Neumonía adquirida en la comunidad (NAC): Aquella que representa las etiologías prevalentes en la comunidad, lo que excluye a pacientes que han estado internados recientemente, en un período, arbitrariamente definido, inferior a 60 días.

\section{Epidemiología}

La neumonía bacteriana ha disminuido paulatinamente su frecuencia en nuestra comunidad, lo que se refleja en una disminución de las internaciones por neumonías de estas etiologías. Hoy en día predominan las neumonías de etiología viral, lo que es manifiesto principalmente en lactantes y pre-escolares.

\section{Etiologías posibles de la neumonía bacteriana}

Se describen (ver párrafo sobre Documentación etiológica) las siguientes:
'Hospital Luis Calvo Mackenna.
Santiago, Chile.
${ }^{2}$ Hospital San Juan de Dios.
Santiago, Chile.
${ }^{3}$ Pontificia Universidad Católica de
Chile. Santiago, Chile.
${ }^{4}$ Clínica Alemana. Santiago, Chile.

Recibido: 29 de abril de 2019

Correspondencia a: José Cofré Guerra pepecofre@gmail.com 
- Streptococcus pneumoniae, en lactantes y preescolares, es la primera etiología, generalmente como sobre-infección de procesos inicialmente de causa viral. La vacunación del lactante (vacunas con 10 y 13 serotipos) ha disminuido la infección neumocóccica invasora, pese a los cual, esta especie continúa siendo considerada la primera etiología de NAC bacteriana en pediatría. Se describen sobre 90 serotipos de $S$. pneumoniae existiendo escasa información de cuáles predominan en la producción de neumonías.

- Haemophilus influenzae serotipo b, propia del lactante; hoy en día, la vacunación programática (2-4-6 y 18 meses de edad) ha disminuido considerablemente la frecuencia de esta etiología.

- Mycoplasma pneumoniae, prevalente en escolares y, con menor frecuencia, en pre-escolares, con un claro comportamiento epidémico.

- Otras especies, de presencia y frecuencia desconocida en nuestro medio, son: Haemophilus influenzae no tipificables o serotipos no b, Streptococcus pyogenes, Chlamydophila pneumoniae y Moraxella catarrhalis.

Staphylococcus aureus no representa, actualmente, una etiología común de NAC en nuestro país, realidad que requiere ser vigilada atentamente.

\section{Patogenia de la infección bacteriana}

Usualmente, la neumonía bacteriana es una infección que proviene de la microbiota presente en el tracto respiratorio superior, precedida y facilitada por una infección respiratoria viral. Alrededor de $95 \%$ de los lactantes son colonizados, transitoriamente, por períodos de tiempo variables, por $S$. pneumoniae, agente que adquiere de sus pares, en el hogar, salas cunas y jardines infantiles. Existe una latencia, medida en un mes, aproximadamente, entre la adquisición de un serotipo de $S$. pneumoniae y el desarrollo de una enfermedad por dicho serotipo. Ello lleva a concebir la infección neumocóccica como una "infección endógena".

Algo similar sucede con las neumonías causadas por $H$. influenzae serotipo b. Este concepto hace una gran diferencia con las neumonías virales que son causadas por agentes exógenos de reciente contagio. Antes de la introducción de la vacuna conjugada anti-Haemophilus influenzae serotipo b (año 1996), 2 a 4\% de los lactantes portaba esta bacteria en su faringe y se documentó su transmisibilidad; hoy, la tasa de portación de $H$. influenzae b, siendo desconocida, se supone mínima.

Ocasionalmente, el origen de una NAC es aspirativo (neonatos) o bacteriémico (paciente con septicemia), condiciones que no se incluyen en estas recomendaciones.

\section{Cuándo una NAC es o puede ser de etiología bacteriana}

Como concepto global, conocer la etiología de una neumonía es complejo (ver párrafo Documentación etiológica); no habiendo una fácil documentación de su etiología, la presunción de que una NAC es de causa bacteriana se basa en el juicioso análisis conjunto de los componentes clínicos, de laboratorio general, radiológicos y microbiológicos de cada caso.

\section{Criterios clínicos}

La semiología orientadora a una etiología bacteriana puede ser muy categórica (con mayor facilidad y frecuencia se encontrará en el escolar) o muy inespecífica. Es un desafío aún mayor reconocer la sobreinfección bacteriana en el curso de una neumonía viral (particularmente en lactantes).

- Hechos semiológicos propios de una neumonía bacteriana. Fiebre alta $\left(>39^{\circ} \mathrm{C}\right.$ axilar) sostenida durante varios días, compromiso del estado general expresada en palidez, hipoactividad y rechazo de alimentos, quejido respiratorio, aleteo nasal, dolor torácico, herpes labial (en niños mayores) y signos clínicos de consolidación pulmonar (matidez, broncofonía, llanto broncofónico, pectoriloquia, soplo tubario, pectoriloquia áfona, soplo pleurítico). Las crepitaciones indican compromiso alveolar pero no son patognomónicas de una infección bacteriana.

- Hechos semiológicos sugerentes de una sobreinfección bacteriana en una infección respiratoria viral. (Generalmente esto sucede a partir de 72 o más horas de evolución de la infección viral, inusualmente antes). Instalación de fiebre en el curso de un cuadro viral que cursaba afebril (siempre descartar el desarrollo de un foco ótico), mayor decaimiento (palidez, hipoactividad, acentuación de la anorexia, vómitos), incremento de la tos, disnea en ausencia de mayor obstrucción bronquial, aparición de signos de consolidación pulmonar en la auscultación (ver párrafo anterior).

Las infecciones pulmonares causadas por adenovirus suelen tener un comportamiento clínico que se asemeja a una sobreinfección bacteriana instalada en una infección inicialmente viral.

En ocasiones, la semiología en una neumonía bacteriana simula una afección abdominal siendo necesario descartar un abdomen agudo "quirúrgico" o puede, simplemente, presentarse como un episodio febril sin focalización clínica.

\section{Parámetros inflamatorios}

Reactantes de fase aguda clásicos descritos como pro- 
pios de una infección bacteriana son la leucocitosis (según edad), neutrofilia y proteína $\mathrm{C}$ reactiva (PCR) elevada.

- Leucocitosis: es inespecífica, pues su elevación se observa también en situación de estrés y corticoterapia en curso. Algunas etiologías virales como adenovirus e influenza estimulan la leucocitosis. Valores superiores a $20.000-30.000$ leucocitos $/ \mathrm{mm}^{3}$ podrían tener un mayor valor predictor de infección bacteriana.

- Neutrofilia: orientador hacia una etiología bacteriana es un valor porcentual de leucocitos segmentados $>70 \%$, aunque también es inespecífico.

- PCR: como los anteriores, ha sido difícil, en ausencia de un estándar de oro para definir una etiología bacteriana, recomendar un valor de corte sobre el cual la PCR apoye la etiología bacteriana. En afecciones como sarampión, se ha definido un valor de corte superior a $90 \mathrm{mg} / \mathrm{L}$ como indicativo de infección/ sobreinfección bacteriana. Sin embargo, es frecuente observar valores superiores a $100 \mathrm{mg} / \mathrm{L}$ como único parámetro sugerente de neumonía bacteriana, en pacientes con adenovirus o influenza. En definitiva, mientras más elevada sea la PCR, por sobre 90 $\mathrm{mg} / \mathrm{L}$, la probabilidad de una infección bacteriana es mayor.

En consecuencia, los parámetros inflamatorios señalados han de ser interpretados en conjunto con el aspecto clínico del paciente y los hallazgos radiológicos que se describen a continuación.

\section{Criterios radiológicos}

Evaluaciones protocolizadas efectuadas entre expertos para la interpretación de las imágenes radiológicas de tórax, en pacientes con infección respiratoria baja, llevaron a dos conclusiones fundamentales:

- Que establecer la naturaleza bacteriana de una NAC a través de imágenes radiológicas es sumamente difícil, en especial, en el caso de infecciones virales sobreinfectadas

- Que las descripciones de los hallazgos y las interpretaciones de los mismos, son usualmente variables entre radiólogos (lo que también se observa entre los médicos tratantes).

Imágenes altamente sugerentes de una etiología bacteriana son: consolidación lobar o segmentaria, única o múltiple, efusión pleural, abscedación. Los signos radiológicos mencionados son más frecuentes de hallar en escolares. Hoy en día, la primera causa de supuración pleuro-pulmonar es $S$. pneumoniae, toda vez que $S$. aureus es muy esporádico como causa de neumonías. Las micro-consolidaciones, con tendencia a confluir, tienen un menor valor en discriminar entre infección de etiología bacteriana vs viral.
La complejidad interpretativa es mayor en lactantes que cursan con una infección pulmonar viral, cuando se sospecha de una sobreinfección bacteriana. Más aún, las neumonías causadas por adenovirus, suelen evolucionar con imágenes de consolidación difusas y efusión pleural.

La neumonía causada por $M$. pneumoniae y C. pneumoniae suele combinar imágenes radiológicas propias de neumonía viral (hiperinsuflación, imágenes intersticiales) con imágenes de consolidación pulmonar, de límites difusos y en número de una o más, e incluso, a veces bilaterales.

\section{Manifestaciones clínicas y parámetros de laboratorio sugerentes de infección por Mycoplasma pneumoniae}

\section{Epidemiología}

Dos hechos epidemiológicos son cardinales para sospechar una enfermedad causada por M. pneumoniae: la existencia de un brote epidémico de esta etiología y la edad del paciente. Básicamente, M. pneumoniae es causa de neumonía en escolares y pre-escolares; a menor edad se le ha relacionado con infecciones respiratorias altas. Un tercer elemento orientador puede ser el contacto, dos o tres semanas antes, con un caso índice confirmado o sospechoso de esta afección.

\section{Manifestación clínica}

Afección respiratoria afebril o escasamente febril, caracterizada por tos persistente, productiva o irritativa, a veces con signos de obstrucción bronquial, en un paciente sin antecedente de asma ni síndrome bronquial obstructivo recurrente, sumado a signos auscultatorios de neumonía y evolución refractaria al uso de $\beta$-lactámicos. Puede acompañarse de exantemas de las más variadas formas e intensidades (10-15\% de los casos). Con escasa frecuencia determina falla respiratoria exigiendo el apoyo con oxígeno y kinesiterapia respiratoria (= hospitalización).

\section{Imagenología}

La Rx de tórax muestra signos de hiperinsuflación, asociada a consolidación(es) difusa(s) y atelectasia(s).

\section{Laboratorio general}

Moderada leucocitosis, ocasionalmente anemia hemolítica, PCR y VHS en valores intermedios o normales.

Mycoplasma pneumoniae y $C$. pneumoniae tienen un comportamiento clínico y ante el laboratorio similar entre ellos, características por las que se les denomina "atípicos". 


\section{Documentación etiológica de una neumonía adquirida en la comunidad}

\section{Secreciones respiratorias}

La expectoración en niños está expuesta a su contaminación por la microbiota existente en el tracto respiratorio superior $\mathrm{y}$, por ende, no se recomienda su estudio, como sí se practica en adultos. Lo mismo sucede con el estudio de secreciones respiratorias obtenidas con hisopados o aspirados nasofaríngeos en lactantes y pre-escolares. Por otra parte, una muestra de secreción traqueo-bronquial obtenida al momento de intubar un paciente con NAC, recién ingresado en una Unidad de Cuidados Intensivos, sí tiene un valor orientador hacia la etiología del proceso neumónico.

Específicamente, ante la sospecha de M. pneumoniae, puede obtenerse una muestra de expectoración (mejor que el hisopado o aspirado naso-faríngeo) con asistencia de kinesiólogo, para estudio etiológico mediante técnicas de amplificación genómica (reacción de polimerasa en cadena-RPC).

\section{Hemocultivo}

El hallazgo en hemocultivo (1 o más) de alguno de los patógenos respiratorios arriba descritos, tiene valor etiológico; el rendimiento de este examen es bajo ( $~ 5-10 \%)$, recomendándose su obtención, antes de iniciar antibioterapia en un paciente que ha de ser internado, si la presunción de una etiología bacteriana es alta. El máximo rendimiento del examen se obtiene con una serie de dos hemocultivos obtenidos por veno-punciones diferentes. El volumen de sangre a obtener para optimizar el rendimiento del hemocultivo es de:

Lactantes: 2-4 cc por muestra.

Pre-escolares (12-18 kg): 3-10 cc por muestra.

Escolares: (18-30 kg): $10 \mathrm{cc}$ por muestra.

Adolescentes (sobre $30 \mathrm{~kg}$ ): 10-20 cc por muestra.

\section{Líquido pleural}

En todo paciente que es internado por una NAC y que presenta una efusión pleural que se estima radiológicamente susceptible de ser puncionada con éxito, se recomienda efectuar una punción pleural diagnóstica para pesquisa etiológica (aun cuando no hubiera la indicación de efectuar un drenaje de la efusión). El líquido obtenido debe ser vertido en: un frasco de hemocultivo y, a la vez, en un tubo estéril seco, para efectuar una tinción de Gram directa e, idealmente, debe ser enviado a estudio por biología molecular (RPC para bacterias).

\section{Punción pulmonar}

Método dejado de lado en la práctica clínica por su naturaleza invasora, aunque la experiencia en su uso indicó que es bien tolerado por el paciente, con escasa fre- cuencia de complicaciones. Ha servido para acercarse al conocimiento de la etiología bacteriana de las neumonías en diversas latitudes -su rendimiento mediante cultivo bacteriológico es cercano a 50\%-aunque las publicaciones más recientes datan ya de 15 años o más (era pre-vacunal). Es un método aplicado también en necropsias.

\section{Orina}

La pesquisa de antígenos neumocóccicos en orina no tiene en niños la utilidad clínica descrita en adultos, debido al alto porcentaje de portación nasofaríngea de la bacteria, que arroja falsos positivos.

\section{Diagnóstico de neumonía por Mycoplasma pneumoniae}

La detección de IgM específica es el método más difundido en la comunidad, aunque su sensibilidad y especificidad son deficientes en comparación con el diagnóstico por cultivo y por RPC. Su defecto mayor es tener falsos positivos por persistir los títulos de IgM elevados durante varios meses tras una infección aguda. Hoy en día, se ha introducido el diagnóstico por amplificación de ADN específico, en secreción bronquial, aunque aún, está escasamente disponible en laboratorios asistenciales.

\section{Tratamiento antimicrobiano de la neumonía adquirida en la comunidad}

Considerando las etiologías presuntas principales de $\mathrm{NAC}$, los fármacos de elección se mencionan y fundamentan a continuación:

\section{Neumonía presunta o confirmadamente neumocóccica}

- Terapia oral: amoxicilina. Su acción anti-neumocóccica es superior a macrólidos/azálidas y cefalosporinas orales de primera, segunda y tercera generación.

\section{Dosificaciones y frecuencia de administración}

La siguiente recomendación está basada en (apoyo bibliográfico en el listado de referencias):

- La redefinición, efectuada por el CLSI en el año 2008, de puntos de corte para establecer in vitro susceptibilidad y resistencia de $S$. pneumoniae a penicilina y amoxicilina

- Datos recientes (años 2011-2017) de la sensibilidad in vitro de $S$. pneumoniae en nuestro medio ( 0 a $1 \%$ de resistencia a penicilina en procesos bacteriémicos incluyendo neumonías), estudio efectuado por el ISP.

- Estudios de la farmacocinética de amoxicilina en diversas dosificaciones y frecuencias de administración en lactantes, efectuados por Fonseca y cols. en 
Brasil, demostrando que amoxicilina dos veces al día se comporta desde punto de vista PK, en exactamente igual que tres veces al día.

- El conocimiento que amoxicilina es más activa que penicilina G sódica sobre S. pneumoniae, como fuera comprobado en nuestro medio por Rosenblut y cols.

- Estudios de campo efectuados en niños con neumonía, sobre eficacia comparativa de distintas dosificaciones y frecuencias de administración de amoxicilina. Estudios en Brasil y Pakistán así lo han comprobado.

- Recomendaciones entregadas por Johns Hopkins University, Maryland, E.U.A. 2015 (Harriet-Lane Handbook) para comunidades con escasa resistencia de $S$. pneumoniae a penicilina.

- Recomendaciones entregadas en las Guías para el tratamiento de la neumonía adquirida en la comunidad finlandesa, en circunstancias de baja resistencia de $S$. pneumoniae a penicilina.

- Guía elaborada en Calgary, Alberta, Canadá, para prescribir dosis bajas de amoxicilina en infecciones neumocóccicas, en niños y adultos, considerando el perfil de susceptibilidad que han observado en su comunidad durante los últimos 10 años.

- Recomendaciones conjuntas de la Academia Americana de Pediatría y la Internacional Society of Infectious Diseases para el tratmiento de NAC en niños en comunidades con baja frecuencia de resistencia a penicilina.

- Reciente $31^{\circ}$ versión de Redbook 2018-21.

Amoxicilina: $50 \mathrm{mg} / \mathrm{kg} /$ día fraccionada cada $12 \mathrm{~h}$, durante 7 dias, respuesta terapéutica que debe ser evaluada en 48 h desde su inicio.

La resistencia in vitro de $S$. pneumoniae a macrólidos, según el informe de vigilancia del ISP arriba mencionado, alcanza a alrededor de $30 \%$ en general y casi $50 \%$ en pacientes bajo 5 años de edad. Poderoso argumento para no considerar esta alternativa terapéutica en NAC para lactantes ni para pacientes mayores con presunción de etiología neumocóccica.

- Terapia parenteral: penicilina G sódica (100.00 UI/kg/ día fraccionada cada $6 \mathrm{~h}$ ) y ampicilina ( $100 \mathrm{mg} / \mathrm{kg} / \mathrm{día}$, fraccionada cada $6 \mathrm{~h}$ ). Este segundo tiene la ventaja de cubrir el espectro de las infecciones por Haemophilus influenzae (no productores de $\beta$-lactamasa).

- La respuesta terapéutica que debe ser evaluada en $48 \mathrm{~h}$ desde su inicio.

- Cefalosporinas de tercera generación parenterales son una indicación de excepción, que debe ser discutida, caso a caso, con el especialista infectólogo.

La terapia antimicrobiana señalada no cubre otras etiologías como $H$. influenzae productor de $\beta$-lactamasa (capsulado y no tipificable) ni $M$. catarrhalis, ambas etiología, de baja incidencia como para considerar su cobertura en un esquema antimicrobiano empírico inicial.

\section{Neumonía atípica, presuntamente causada por Mycoplasma pneumoniae o Chlamydophila pneumoniae}

La indicación de macrólidos/azálidas con la presunción etiológica de $M$. pneumoniae o C. pneumoniae es de beneficio incierto, según un meta-análisis del año 2015 , por falta de estudios bien diseñados. Se recomienda prescribir antimicrobiano en el paciente con neumonía que cursa con compromiso de su estado general y compromiso funcional respiratorio que requiere hospitalización. La bronquitis sugerente de infección por $M$. pneumoniae, probablemente no se beneficie de una antibioterapia.

Fármaco de elección: azitromicina, por su facilidad de administración, esquema acortado y tolerancia digestiva, que favorecen una mejor adherencia al tratamiento en comparación con macrólidos. Dosificación: $10 \mathrm{mg} / \mathrm{kg}$ una vez al día, durante 5 días.

El uso desmedido de macrólidos/azálidas en una neumonía, con la presunción de ser causada por patógenos "atípicos", amenaza con favorecer el surgimiento de resistencia a ellos, riesgo que no es vigilado en nuestro medio, pero sí es una realidad presente en otras latitudes donde se describe hasta $70 \%$ de resistencia in vitro de $M$. pneumoniae a macrólidos/azálidas.

Prevención de casos secundarios en caso de infección por M. pneumoniae. Se justifica la quimioprofilaxis secundaria por contacto sostenido y cercano con un caso índice si:

- El hospedero contacto adolece de una capacidad funcional cardio-respiratoria disminuida o tiene una condición de asmático.

- Existe un segundo caso en el seno familiar o en instituciones cerradas (internados, hogares de menores, colegios).

\section{Conceptos fundamentales}

- La NAC bacteriana en pediatría obedece a dos etiologías principales: Streptococcus pneumoniae (a toda edad) y Mycoplasma pneumoniae (en escolares y pre-escolares). Ocasionalmente se detectan otras etiologías, entre ellas Haemophilus influenzae no tipificables y Streptococcus pyogenes.

- Staphylococcus aureus es considerado actualmente un patógeno asociado a neumonías nosocomiales.

- El diagnóstico de neumonía presuntamente bacteriana, más frecuente y fácil de plantear en escolares que en lactantes, debe surgir del juicioso análisis semiológico, imagenológico y de los exámenes generales, en cada paciente. 
- En Chile, la vigilancia nacional de laboratorio, radicada en el Instituto de Salud Pública, señala la existencia de mínima resistencia in vitro de $S$. pneumoniae a penicilina $(0-1,0 \%$ en los últimos 5 años) para procesos localizados fuera del sistema nervioso central.

- El tratamiento de elección para la NAC de manejo ambulatorio, a toda edad superior a 3 meses, es amoxicilina.

- De acuerdo a los resultados de la vigilancia de susceptibilidad in vitro de $S$. pneumoniae en nuestro medio, las dosis recomendables de amoxicilina para el tratamiento empírico inicial de la NAC de manejo ambulatorio son de $50 \mathrm{mg} / \mathrm{kg} /$ día, fraccionada en dos tomas diarias.

- El tratamiento de elección inicial para la NAC de manejo hospitalario, a toda edad superior a 3 meses, es penicilina o ampicilina intravenosa.

- De acuerdo a los resultados de la vigilancia de susceptibilidad in vitro de $S$. pneumoniae en nuestro medio, las dosis recomendables de penicilina $G$ sódica y ampicilina ev para tratamiento empírico inicial de la NAC en pacientes que se hospitalizan son de $100.000 \mathrm{UI} / \mathrm{kg} /$ día y $100 \mathrm{mg} / \mathrm{kg} /$ día, respectivamente, fraccionado cada $6 \mathrm{~h}$.

- En la NAC causada por, o presuntamente debida a $M$. pneumoniae el tratamiento antimicrobiano recomendado es azitromicina, por su mejor tolerancia, frecuencia de administración y posibilidad de adherencia a la terapia.

\section{Lecturas recomendadas}

\section{Etiología de la neumonía en pediatría}

- Schuster A, Pino M, Neira M, Vildósola C, Faini L. La punción biopsia pulmonar como método diagnóstico de las neumopatías de la infancia. Pediatría (Santiago) 1966; 9: 9-12.

- Mimica I, Donoso E, Howard J E, Ledermann GW. Lung puncture in the etiological diagnosis of pneumonia: a study of 543 infants and children. Am J Dis Child 1971; 122: 278-82. PMID: 4398908.

- Vuori-Holopainen E, Peltola H. Reappraisal of lung tap: Review of an old method for better etiologic diagnosis of childhood pneumonia. Clin Infect Dis 2001; 32: 715-26. doi: 10.1086/319213

- Vuori-Holopainen E, Salo E, Saxén H, Hedman K, Hyypiä T, Lahdenperä R, et al. Etiological diagnosis of childhood pneumonia by use of transthoracic needle aspiration and modern microbiological methods. Clin Infect Dis 2002; 34 (5): 583-90. Epub 2002 Jan 16. doi: $10.1086 / 338642$.
- Tramper-Stranders G A. Childhood community-acquired pneumonia: A review of etiology-and antimicrobial treatment studies. Paediatr Respir Rev 2018; 26:41-48. doi: 10.1016/j.prrv.2017.06.013.

\section{Herramientas de diagnóstico}

- Cherian T, Mulholland E K, Carlin J B, Ostensen H, Amin R, de Campo M, et al. Standardized interpretation of paediatric chest radiographs for the diagnosis of pneumonia in epidemiological studies. Bull World Health Org 2005; 83 (5): 353-9. doi: S004296862005000500011.

- Neuman M I, Hall M, Lipsett S C, Hersh A L, Williams D J, Gerber J S. Utility of blood culture among children hospitalized with community-acquired pneumonia. Pediatrics 2017; 140 (3): 1. doi: 10.1542/peds.2017-1013.

\section{Resistencia de Streptococcus pneumoniae a antimicrobianos}

- Friedland I R, Klugman K P. Antibiotic-resistant pneumococcal disease in South African children. Am J Dis Child 1992; 146: 920-3. doi: 10.1001/archpedi.1992.02160200042023.

- Yu V L, Chiou C C, Feldman C, Ortqvist A, Rello J, Morris A J, et al. An international prospective study of pneumococcal bacteremia: correlation with in vitro resistance, antibiotics administered, and clinical outcome. International Pneumococcal Study Group. Clin Infect Dis 2003; 37: 230-7. doi: 10.1086/377534.

- Rosenblut A, Santolaya M E, González P, Borel C, Cofré J. Penicillin resistance is not extrapolable to amoxicillin resistance in Streptococcus pneumoniae isolated from middle ear fluid in children with acute otitis media. Annals Otol Rhinol Laryngol 2006; 115; (3): 186-90. doi: 10.1177/000348940611500305.

- Clinical Laboratory Standards Institute (CLSI). Performance Standards for Antimicrobial Susceptibility Testing; Eighteenth Informational Supplement. January 2008. M100-S18. Vol $8 \mathrm{~N}^{\circ} 1$. Replaces M100-S17. Vol $27 \mathrm{~N}^{\circ} 1$.

- Weinstein M P, Klugman K P, Jones R N. Rationale for revised penicillin susceptibility breakpoints versus Streptococcus pneumoniae: coping with antimicrobial susceptibility in an era of resistance. Clin Infect Dis 2009; 48: 1596-600. doi: 10.1086/598975.

- Instituto de Salud Pública. Vigilancia de Laboratorio de Streptococcus pneumoniae procedente de enfermedad invasora. 2011-2017. Boletín de Vigilancia de Laboratorio 2018; 8 (3): 1-19 http://www.ispch.cl/ sites/default/files/BoletinNeumo-08012019B\%20(1). pdf.

- Instituto de Salud Pública de Chile. Ministerio de Salud. Programa de Control de Infecciones Asociadas a la Atención de Salud. Boletín de Resistencia Anti- 
microbiana. Chile 2018. http://www.ispch.cl/sites/ default/files/BoletinRam2018-08012019A.pdf.

\section{Evidencia que apoya la recomendación de amoxicilina en dosis de $50 \mathrm{mg} / \mathrm{kg} / \mathrm{peso} / \mathrm{día}$ fraccionada cada $12 \mathrm{~h}$}

- Tabish Hazir, Shamim A Qazi, Yasir Bin Nisar, Sajid Maqbool, Rai Asghar, Imran Iqbal, et al. Comparison of standard versus double dose of amoxicillin in the treatment of non-severe pneumonia in children aged 2- 59 months: a multi-centre, double blind, randomised controlled trial in Pakistan. Arch Dis Child 2007; 92: 291-7. doi: 10.1136/adc.2005.092494.

- Daschner F D, Behre U, Dalhoff A. Prospective clinical trial on the efficacy of amoxicillin administered twice or four times daily in children with respiratory tract infections. J Int Med Res 1981; 9: 274-6. DOI: 10.1177/030006058100900407.

- Fonseca W, Hoppu K, Rey LC, Amaral J, Qaziet S. Comparing pharmacokinetics of amoxicillin given twice or three times per day to children older than 3 months with pneumonia. Antimicrob Agents Chemother 2003; 47: 997-1001. DOI: 10.1128/ AAC.47.3.997-1001.2003.

- Vilas-Boas A L, Fontoura M S H, Xavier-Souza G, Araújo-Neto C A, Andrade S C, Brim R V, et al. Comparison of oral amoxicillin given thrice or twice daily to children between 2 and 59 months old with non-severe pneumonia: a randomized controlled trial. Antimicrob Chemother 2014; 69: 1954-9. doi:10.1093/jac/dku070.

- Vilas-Boas A L, Nascimento-Carvalho C M on behalf of the PNEUMOPAC-Efficacy Study Group. Comparison of oral amoxicillin given thrice or twice daily to children between 2 and 59 months old with non-severe pneumonia: a randomized controlled trial-authors' response. J Antimicrob Chemother 2015; 70 (2): 636-8, https://doi.org/10.1093/jac/dku440.

- Patel A, Dhande L, Bang A, Malik A, Chelliah L R, et al. Efficacy of 7 day course of ambulatory oral amoxicillin for treating severe pneumonia in 3-59 months old Indian children - multicentric randomised control trial (ISPOT Group). Pediatric Research 2011; 70: 15. doi: 10.1203/01.pdr.0000403893.61640.b6.

- Addo-Yobo E, Anh D D, El-Sayed H F, et al. Outpatient treatment of children with severe pneumonia with oral amoxicillin in four countries: the MASS study. Trop Med Int Health 2011; 16: 995-1006. doi: 10.1111/j.1365-3156.2011.02787.x.

- Bari A, Sadruddin S, Khan A, et al. Community case management of severe pneumonia with oral amoxicillin in children aged 2-59 months in Haripur district, Pakistan: a cluster randomised trial. Lancet 2011; 378: 1796-803. doi: 10.1016/S0140-6736(11)61140-9.

- Archana B. Patel, Akash Bang, Meenu Singh, Leena
Dhande, Luke Ravi Chelliah, et al, and ISPOT Study Group. A randomized controlled trial of hospital versus home based therapy with oral amoxicillin for severe pneumonia in children aged $3-59$ months: The IndiaCLEN Severe Pneumonia Oral Therapy (ISPOT) Study- BMC Pediatrics (2015) 15: 186 doi: 10.1186/ s12887-015-0510-9.

- Tramper-Stranders G A. Childhood community-acquired pneumonia: A review of etiology-and antimicrobial treatment studies. Paediatr Respir Rev 2018; 26:41-48. doi: 10.1016/j.prrv.2017.06.013.

\section{Recomendaciones entregadas por fuentes internacionales seleccionadas}

- Grant G B, Campbell H, S F Dowell, Graham S M, Klugman K P, Mulholland E K, et al. Recommendations for treatment of childhood non-severe pneumonia. Lancet Infect Dis 2009; 9: 185-96. doi: 10.1016/ S1473-3099(09)70044-1.

- Rajapakse N S, Vayalumkal J V, Vanderkooi O G, Ricketson L J, Kellner J D. Time to reconsider routine high-dose amoxicillin for community-acquired pneumonia in all Canadian children. Paediatr Child Health 2016; 21 (2) March: 65-6. https://www.ncbi.nlm.nih. gov/pmc/articles/PMC4807794/pdf/pch-21-65.pdf.

- Terhi Tapiainen, Janne Aittoniemi, Johanna Immonen, Heli Jylkk€a, Tuula Meinander, Kirsi Nuolivirta et al. Finnish guidelines for the treatment of community-acquired pneumonia. Acta Pædiatrica 2016; 105 (1): 39-43. doi:10.1111/apa.13177.

- American Academy of Pediatrics. Pneumococcal Infections. Kimberly DW, Brady MT, Jackson MA, Long SS, eds. Red Book 2018 Report of the Committee on Infectious Diseases. 31st ed. Itasca IL. American Academy of Pediatrics 2018. p: 639-51.

- The Harriet Lane Handbook 2018. A Manual for Pediatric House Officers. Twenty First Edition. Helen K. Hughes \& Lauren K. Kahl, eds. Elsevier. https:// ia800100.us.archive.org/21/items/HarrietLane/harriet $\% 201$ lane.pdf.

- Guideline for the diagnosis and management of community acquired pneumonia: pediatrics. 2008 Update. Administered by the Alberta Medical Association.

- Bradley J S, Byington C L, Shah S S, Alverson B, Carter E R, Harrison C, et al. The management of community-acquired pneumonia in infants and children older than 3 months of age: clinical practice guidelines by the Pediatric Infectious Diseases Society and the Infectious Diseases Society of America. Clin Infect Dis 2011; 53 (7): e25-76. doi: 10.1093/cid/cir531.

- Diretrizes Brasileiras em neumonía adquirida na comunidade em pediatría 2007. J Bras Pneumol 2007; 33 (Supl 1): S31-S50. http://www.scielo.br/pdf/jbpneu/ v33s1/02.pdf. 
- Nascimento-Carvalho C M. Pharmacotherapy of childhood pneumonia. Exp. Opin Pharmacother 2010; 11 (2): 225-31. doi: 10.1517/14656560903433714.

- Guía de práctica clínica en el tratamiento del niño con neumonía adquirida en la comunidad. Guía Colombiana 2010. Posada A, Parra W, eds. p 37, p 67. http:// portal.neumopediatriacolombia.com/wp-content/ uploads/2017/03/Neumonia.pdf.

- Das R R, Singh M. Treatment of severe community-acquired pneumonia with oral amoxicillin in under-five children in developing country: a systematic review. PLoS ONE. 2013;8, e66232. doi: 10.1371/journal. pone.0066232.

- Tramper-Stranders G A. Childhood community-ac- quired pneumonia: A review of etiology- and antimicrobial treatment studies. Paediatr Respir Rev 2018; 26:41-48. doi: 10.1016/j.prrv.2017.06.013.

- Gardiner S J, Gavranich J B, Chang A B. Antibiotics for community-acquired lower respiratory tract infections secondary to Mycoplasma pneumoniae in children. Cochrane Database of Systematic Reviews 2015, Issue 1. Art. No.: CD004875. doi: 10.1002/14651858. CD004875.pub5.

- Lee H, Yun K W, Lee H J, Choi E H. Antimicrobial therapy of macrolide-reststant Mycoplasma pneumoniae pneumonia in children. Expert Rev Anti Infect Ther 2018; 16 (1): 23-34. doi: 10.1080/14787210.20 18.1414599 . 